Hyperesthesia was marked throughout. Retraction of but was soon discontinued, as it seemed to increase the head became so marked that deglutition became at hyperesthesia and general symptoms of neuritis. We times very difficult or impossible. There was extreme think this remedy, if given at all, should be given opisthotonos, which, with the retraction of the head, cautiously and not so long as symptoms of neuritis continued for fully five weeks. His arms were flexed are at all marked.

on the abdomen or chest, the lower limbs drawn up and flexed at the knees. The Kernig symptom was present for some time, but later any attempt at extension became painful. He lay on his side in the manner indicated and, to take nourishment, with some assistance, he would turn on his abdomen, which would throw the retracted head in an upright position so that he could receive it in a fairly natural manner. In assuming this position, however, he never failed to implore his attendant to be careful.
There was deafness and intolerance of light. His pulse during the first week ranged from 100 to 120 per minute, but during the second week it dropped to from 55 to 70 (mostly beating about 60 ) where it remained for some weeks, then it again averaged about 90 , and so remained until he was discharged. Temperature for first thirty-six hours was from 100 to 103. After that seldom above 101, and ranged mostly from 99 to 100 . At times it was subnormal. Respiration was from 20 to 30 , mostly about 24 per minute. During the second, third and fourth weeks it was markedly Cheyne-Stokes in character, the period of apnea lasting as long as twenty-five seconds. The pulse was also much influenced in its rhy thm and volume during these changes in the respiration. The Cheyne-Stokes phenomenon was most marked during a period of albuminuria lasting several days, during the fourth week. Twice he had an attack of dyspnea with violent, painful constriction in the chest. His face had the anxious expression of one suffocating, while he tore wildily at the clothes about the chest. The urine was examined in both cases every day or two, both chemically and microscopically. The boy's urine was frequently abundant, with low specific gravity, $100 \tilde{s}$ alkaline, with excess of triple phosphates. At times, however, it was scanty, with high specific gravity, 1025. The father's urine was generally fairly concentrated.

Both these cases received morphin and the boy took, during his illness, very much more than his father. At times the boy required 0.016 gram every four hours to control the pain. It was, however, given mostly in 0.008 gram duses every two hours, but while giving it this way an extra dose was required at times. The morphin was only given as required, and as he began to improve intervals of half a day to a day would elapse in which it was not necessary to give morphin, but when the pain did recur it would yield to nothing short of the full dose. Even the very last paroxysm of pain required no less than three doses of the morphin. Atropia was always given with the morphin but not increased with it. Small doses of bromid were given throughout the illness. With the large doses of morphin required by the boy, his desire for nourishment did not seem impaired, and it is worthy of note that he took an average of a third more milk than his father, taking seldom less than a quart per day. With a view of promoting gastric digestion dilute hydrochloric acid was given in small doses. It did not, however, improve the digestion, but seemed to aggravate the general symptoms. The potassii acetas, on the other hand, seemed to have a favorable influence. Fowler's solution, in small doses, was tried as a reconstructive after convalescence was established,

February 25 another boy, aged 15 , became sick with symptoms of the disease, but they were mild, and in a week he was again feeling quite well. Two other members of the family, some days later, became sick and were indisposed for several days, but the symp. toms did not point sufficiently to the disease to include them. There was, however, one other case in another family, a little girl 8 years old, who was sick fourteen days. Early symptoms were the same as those of cases described. There was, however, in this case, marked dysenteric discharges containing mucus, pus and blood. The case was also markedly intermittent in type. The pain was controlled with acetanilid, no opiate being given. Bromid was given in small quantity.

All these cases were frequently and carefully examined for signs of pulmonary changes, but with negative results, at least so far as physical signs were concerned. The disturbed or slightly increased respiration at times noted was due either to disturbed central innervation or peripheral nerve involvement making normal respiration painful, and hence the more frequent but shallow respirations.

\title{
OBSERVATIONS UPON THE SPECIFIC TREATMENT OF TUBERCULOSIS.
}

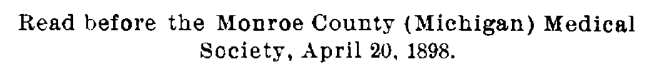

Briefly, today the pathology of tuberculosis takes into consideration but one real causative factor, the bacillus. Yet apart from this assumption I can not disabuse my mind of the impression that we may and do have a form of phthisis in which this form of bacillus does not directly figure. Such cases are characterized by a low form of inflammatory action, a fibrosis, if you please, with little or no fever. The expectoration is mucopurulent and profuse; consolidation, usually at the base, is present, with the usual. classic symptoms. I have made many examinations of the sputum of these cases, finding no bacilli. I take it that this class of cases constitutes the longdrawn-out ones, lasting perhaps for years. They are doubtless susceptible to true tubercular infection, which infection usually runs to a rapid termination. The comparative absence of fever and the failure to find the bacilli, point quite conclusively to this form of consumption. If, then, we have among us this separate type of disease, we perhaps have an explanation of some failures along the lines of specific treat. ment.

Since the discovery of the bacilli of tuberculosis by Koch, in 1882, there has been a renewed impulse along various lines of investigation, with a view to find some means or remedy inimical to the growth of this bacillus.

If we stop to consider the fearful mortality of consumption we shall see how eminently humane and just are the efforts to mitigate, cure or control this terrible scourge. The true physician will strive to aid in this conquest, will try all things and hold fast to that which is good. In the use-of Koch's crude 
tuberculin, we found for the first time an agent capable of influencing the tubercular process. That this influence was not salutary, I need not tell you. And yet it was, perhaps, but the too powerful impression of the remedy which increased the degenerative processes. Koch's discovery did not, then, prove to be a distinctly curative agency; but it did serve to demonstrate that in tuberoulin we had an agent whose influence upon tuberculosis was specific, and hence has followed the present spirit of investigation by physicians of every country.

In directing attention to a few agents of more or less conceded specific effect, we will confine our remarks to such remedies as have been used hypodermically, because by no other method can we so certainly obtain the real physiologic effect of any medicine. Among the first of these agents we may mention the Shurly-Gibbes treatment, the basis of which was gold and iodin. That these agents were possessed of some virtue I do not doubt; and yet the result has not warranted a very general application. While Drs. Shurly and Gibbes were able to immune monkeys, the remedies occasioned so much pain in their hypodermic use upon patients that it was difficult to properly carry out the indications. Soon after the treatment was placed before the profession, I obtained some very good results. One patient, whose recovery was thought impossible, is alive and in fair health today. Still, as I said, very few would submit to the pain of these injections. Within the past few months my friend, Dr. E. L. Shurly of Detroit, Mich., has found a solvent for iodin which does not occasion much irritation. The solvent in question is the oil of cloves. It has been found that one and one-half grains of iodin can be dissolved in one drachm of oil of cloves. Of this solution ten minims is given. Better still is it to add an equal amount of sterilized olive onl and then give twenty minims. The injection is given once a day deeply into the thigh or muscles of the back. In the use of this remedy my experience, as well as that of Dr. Shurly, has been fairly good. It is quickly absorbed and little or no irritation follows. While the oil of cloves is used simply as a solvent it may exercise some beneficial effect, for this oil has received considerable commendation in the treatment of tuberculosis, given internally.

The use of nuclein in phthisis is founded upon the theory advanced by Metchnikoff, that the white blood corpuscles act as phagocytes in destroying microorganisms. It has been proven beyond doubt that solutions of nuclein increase leucocytosis to a marked degree. At one time it was thought that this increase was only relative, but experiment shows it to have been absolute. I have been able to observe the in. crease within two hours after the exhibition of the remedy. If, therefore, the theory of action were to work ideally, we might expect these leucocytes to act as scavengers and to destroy the tubercle bacilli or impair their infective power. To a certain extent these results do follow its use. Professor Vaughan of Ann Arbor has found the remedy of pretty general utility in early manifestations of the disease. The consensus of opinion indicates that nuclein is deserving of further trial and that more or less benefit fol. lows its use. In my own experience, I have noticed temporary improvement in nearly all cases. This improvement, however, has not been sufficiently permanent to inspire me with much confidence. Still, in properly selected cases, good will undoubtedly result from its use. At present it is placed upon the market in a 5 per cent. solution, which may be given either by the stomach or hypodermically. If given by the mouth it should be upon an empty stomach in from one to two drachm doses three times a day; hypodermically, fifteen to sixty minims, once a day. It must not be inferred that nuclein has any distinctive action upon the tubercle bacilli, but that it acts more or less alike upon all infective germs and should therefore offer some curative aid to their resulting diseases. It is also claimed by Dr. Vaughan to possess direct germicidal action.

Serotherapy.-After the apparent failure of tuberculin, Dr. Maragliano of Genoa devised a serum from the use of which good results are claimed. This serum is supposed to be derived from the horse in a manner somewhat analogous to that of the antitoxin of diphtheria. Dr. Maragliano's method of treatment claims 68 per cent. of improvement. The details of the manufacture and use of this serum are not familiar to the profession of this country, and I have seen no mention of any trial having been made in America. However, Dr. Maragliano's professional reputation is such that his deductions have had no inconsiderable weight with the medical profession of the world.

Dr. P. Paquin of St. Louis, has produced a horse antitubercle serum from the use of which he claims 66 per cent. improvement. Other experimenters have also reported favorably, so that it seems just to accord to these antitoxins a permanent place in the serotherapy of tuberculosis. Both are given hypodermically. Finally, we come to the consideration of the various modified tuberculins. These are so numerous, and the claims based upon their use so diverse, that I can not hope to give anything like an exhaustive review.

Among the more common may be named antiphthisin, tuberculin R., purified tuberculin, watery extract of tubercle bacilli and oxytuberculin. These various tuberculins differ mainly in the amount of organic matter which they severally contain, also in their composition as to the presence of toxin or antitoxin. The use of some of them is followed by a rise of temperature, somewhat characteristic of the originel tuberculin. It is therefore advised to begin with a small dose and increase very gradually. Dr. Karl von Ruck of Asheville, N. C., has used the purified tuberculin in twenty cases with an improvement in 90 per cent. Four cases have made an absolute recovery and five more are rapidly nearing that result. Koch's new tuberculin seems to have failed and the use of antiphthisin has not been followed by very flattering results, due possibly to improper selection of cases. The doses of these preparations are from 0.1 to 3 c.c. No claims are made for advanced cases. Oxytuberculin is a comparatively recent addition to this line and was brought out by Dr. Joseph Hirschfelder of San Francisco, Cal. His first general communication to the profession was made at the meeting of the Aumerican Medical Association in June of last year. Dr. Hirschfelder is a careful and conservative investigator and his deductions are therefore worthy of some consideration. The method of manufacture consists in gradually adding peroxid of hydrogen to true tuberculin and then sterilizing from 120 to 130 hours. By this "oxidation" process the infective power of the bacilli is destroyed and there is left simply the oxytoxin, the exhibition of which is not followed by interference with the temperature curve. If this toxin be added 
to virulent cultures of this germ, growth ceases; und if applied to local tubercular alcerations, the bacilli disappear and the ulcers heal promptly. Dr. Hirschfelder closes an article in the Medical News with these words: "All these tests show conclusively that the oxytuberculin inhibits the growth of the bacillus of tuberculosis. That this is a specific effect and not due to a general antiseptic action, is proved by the facility with which other germs develop in the fluid. From all the statements made I believe I have proved beyond a reasonable doubt that consumption may be cured by the use of oxytuberculin, if the remedy is administered during the early stages of the disease, and that the cure is effected by direct action upon the causative germ."

My own observations in the trial of this remedy are confined to three cases, none of which come under the classification of early. Case 1 was one of galloping phthisis, in a patient whose mother and sister had died of tuberculosis. The disease first declared itself in June, 1897. The area of tubercular infiltration spread with almost the rapidity of pneumonitis and very soon involved the lower and part of the upper lobe of the left lung. The sputa contained large numbers of bacilli. Within a short time a large abscess had formed in the central portion of the lung. Temperature ranged from 101 to 104 degrees, pulse from 110 to 120 . About this stage of the disease I began the hypodermic use of oxytuberculin in doses of 12 c.c. The results were strikingly beneficial; temperature fell, cough decreased, night sweats were slight and at times none, while the appetite improved and the bacilli could no longer be found. We had, however, to deal with the septic complication incident to the abscess, which without doubt will carry the patient off before long. Were it possible to have drained the abscess cavity, I believe this patient might have recovered, for even at this date the right lung remains unimpaired.

Case 2 was of some three years' standing, or rather is of that length of time, during which time the patient had tubercular pleurisy, two tubercular abscesses of the sternum and a small abscess or cavity in the upper lobe of the left lung. The pulse was rapid, 112, cough annoying, expectoration profuse and well stocked with tubercle bacilli. The temperature ran constantly above 100 degrees. After about six weeks' treatment with oxytuberculin the temperature became normal and has so remained and the bacilli have nearly disappeared from the sputa, with general improvement in nutrition and respiratory power. Dulness has diminished to a marked degree.

Case 3 was one of three years' duration. Both lungs were involved, with a small cavity in the left one. The patient was confined to the bed, with high fever, night sweats and constant cough, bacilli numerous, nutrition poor. Under oxytuberculin she has steadily gained in flesh and the appetite and digestion are good. There is no fever, nor has there been for several weeks. She is constantly about the house and attends to many duties. The last examination of spu. tum failed to show but fow bacilli, and these were difficult to find.

If time had permitted, I would have been pleased to have gone into the detaiis regarding these cases, and yet I think I have shown that this oxytuberculin must be possessed of a wonderful (curative) power. None have been absolutely cured, but each case has demonstrated the benefits derived from this Of this number less than ten have been contributed primary tuberculosis is still considerably below fifty.
Of this number less than ten have been contributed

treatment. Not one of the cases could be classified to point pretty certainly to Dr. Hirschfelder's conclu. sion that in early cases this remedy will cure a large majority.

The large dose and consequent expense of the oxytuberculin will probably to a certain extent prevent its general use. It costs $\$ 5$ for three ounces and is given in doses of from 5 to 20 c.c. hypodermically, daily. Thus far I have used 140 ounces. The injection does not cause reaction or more irritation than an equal amount of water would do. I have never had an abscess or a svmptom of one following the injection. If the theory of its action be correct, we have a remedy which will open up a new era in the treatment of tubercular joint diseases; for instead of using iodoform and like antiseptics, we shall simply inject oxytuberculin with possibly absolute results.

In speaking of these agents as specifics it must not be inferred that they offer the means of a certain cure, only so far as the real causative elements are concerned. The pathologic results of the invasion of the tubercular bacilli may remain, and if sufficiently grave, death will result; yet it is to me remarkable that such extensive and rapid repair follows the exhibition of this modified tuberculin. And finally, it seems to me conclusive that, if ever we find a true specific for tuberculosis, it will be among these agents. There are those who maintain that there is no foundation or proof upon which to build our hope for a treatment of tuberculosis by bacterio-therapy. Scientific skepticism is just, if it be predicated upon the correct appreciation of therapeutic deductions. Metaphysically speaking, we do not know the modus operandi of these various tuberculins, but for that matter, do we comprehend the intrinsic or dynamic power of any common remedial agent? Shall we say that quinin does not cure malarial fever by its specific action upon the plasmodium malariæ? Or that vaccine does not inbibit smallpox? These questions and their details must, therefore, be worked out upon lines of scientific investigation and close observation. If in the end we attain the desired result, what more glorious could redound to the medical profession and to the world? And if we fail, it can not be said of us that we considered the object unworthy our efforts.

TUBERCULOSIS OF THE MAMMARY GLAND. Read by title at the meeting of the obio state Medical Society, May 6,1898 .

BY ALBERT H. FREIBERG, M.D.

SURGEON TO THE PRESHYTERIAN HOSPITAL; ORTHOPEDIC SURGEON TO THE CINCINNATI HOSPITAL. CINCINNATI, OHIO.

Until very recent times tuberculosis of the breast has been considered one of the rarest surgical conditions; this is especially true of primary tuberculosis of this gland: Prior to 1880 we find practically no mention of this condition in surgical treatises save by Astley Cooper and Velpeau. Billroth, in his monograph on diseases of the breast, regarding all previous reports on this subject as inaccurate and lacking histologic confirmation, mentions a case of undoubted tuberculosis of the breast found postmortem in a woman who had died of pulmonary and intestinal tuberculosis. Since this time reports of these cases have multiplied, although the total number of authenticated cases of 